Rev. Elev. Méd. vét. Pays trop., 1979, 32 (4) : 325-333.

\title{
La Brucellose bovine en Côte-d'Ivoire Epidémiologie
}

\author{
par E. PILO-MORON $(*)$, F. PIERRE $(* *)$ et J. B. KOUAME $(* * *)$
}

\begin{abstract}
RÉSUMEE
Une vaste enquête épidémiologique effectuée dans pratiquement toutes les régions géographiques de la Côte-d'Ivoire a confirmé l'existence de la brucellose bovine dans l'ensemble du pays, avec 10,8 p. 100 des douze mille trois cent quarante-trois échantillons de sérum de positifs.

Une enquête sérologique parallèle, menée dans la région Nord, où se situe la majorité du cheptel, a montré que 38 p. 100 des huit cent cinquante-neuf vaches examinćes ont eu au moins un avortement imputable à $B$. abortus ce qui représente 3,2 p. 100 du total du cheptel soumis à notre enquête. Dans les troupeaux examinés, la présence d'hygromas est variable. Des liquides de ponction recueillis, il a été possible d'isoler dix-sept souches de $B$. abortus appartenant aux biotypes I et VI.
\end{abstract}

\section{INTRODUCTION}

La brucellose bovine est solidement implantée sur le continent africain. Abstraction faite des pays de l'Afrique du Nord et de ceux constituant l'Afrique australe, la situation pour le reste du continent figure dans la carte $n^{\circ} 1$, au vu des enquêtes effectuées ces dernières années.

La maladie existe en Côte-d'Ivoire, où elle a été mise en évidence par BOHNEL (1) en 1970 à l'occasion d'une enquête sérologique effectuée dans la région de Korhogo, avec soixante-cinq veaux de positifs sur cinq cent cinquantequatre examinés, soit un taux d'infection de 11,7 p. 100 , et huit villages infectés sur les dixneuf prospectés. La même année, vingt-quatre

(*) et (***) Projet FAO/PNUD IVC/71/505, Laboratoire de Pathologie animale de Bingerville, B. P. 206, Bingerville (République de Côte-d'Ivoire).

(**) Projet FAO/PNUD IVC/71/505, Laboratoire de Pathologie animale, Annexe de Korhogo, B. P. 32, Korhogo (République de Côte-d'Ivoire). des sérums en provenance de la région de Bouaké, examinés au Laboratoire National de l'Elevage de Dakar-Hann (6) ont été reconnus positifs pour un taux d'infection de 75 p. 100 . A la suite d'une nouvelle enquête effectuée en 1973 par le Laboratoire de Bouaké, F. COULIBALY (4) signale 23 p. 100 d'animaux infectés sur les deux cent quatre-vingt-un examinés.

Les enquêtes menées en 1972 et 1973 par GIDEL et collab. (5) ont montré que 15,6 p. 100 des bovins de la région de Korhogo, 3,6 p. 100 de la région de Bouaké, et 13,6 des régions d'Odienné et de Man étaient positifs.

Le but de notre enquête a été de compléter celles déjà effectuées, de dresser la carte de la maladie à la lumière des derniers résultats acquis, fin décembre 1977, et de confirmer l'existence de la brucellose par isolement du germe causal, tout cela pour préciser le rôle que cette maladie joue actuellement dans l'élevage bovin de la Côte-d'Ivoire et celui qu'elle peut être appelée à jouer dans les programmes de développement du cheptel, en cours et à venir. 


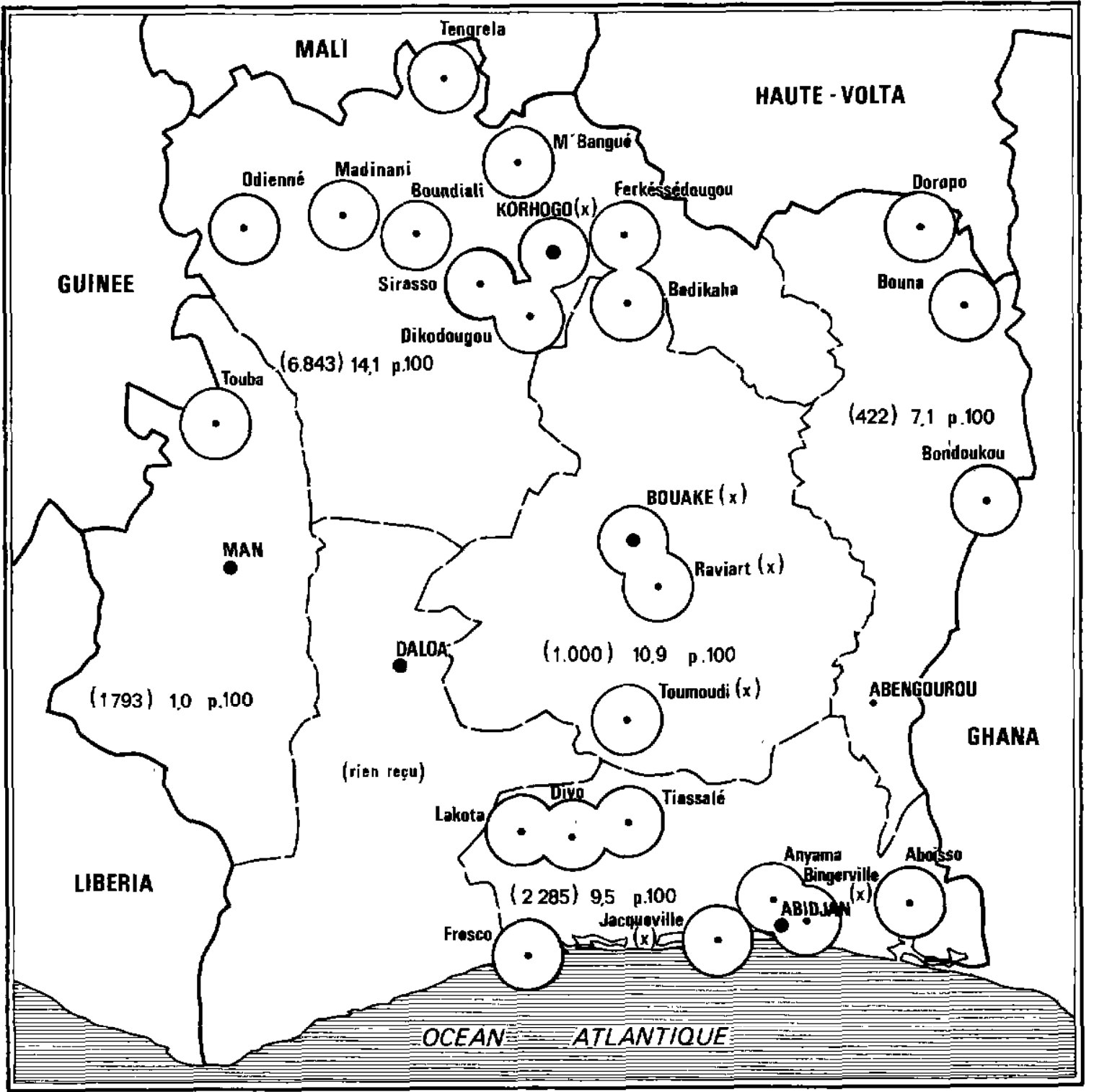

I. SITUATION ACTUELLE DE L'ÉLEVAgE BOVIN. PROGRAMMES DE DEVELOPPEMENT

Actuellement la Côte-d'Ivoire compte environ quatre cent cinquante-huit mille têtes de bovins répartis de la façon suivante :

- Elevage taurin encadré du Nord 225000

— Elevage zébu du Nord ........ 153000

- Ranches et élevage sous palmeraies................ 10000

— Centres d'élevage divers...... 70000

Les fermes d'élevage (ranches, noyaux d'élevage, etc.) ont pour objectif à moyen et à long terme de répondre aux demandes croissantes en viande de boucherie. Pour cela, le Gouvernement mène un vaste plan de développement $\mathrm{du}$ cheptel qui se traduit:

A) dans le nord du pays (régions de Korhogo, Boundiali...), qui est le plus riche en effectif bovin, par deux opérations, en cours :

a) l'opération "Elevage taurin du Nord", qui vise à la mise en valeur rationnelle de la zone dense de Korhogo en augmentant le plus rapidement possible les effectifs du cheptel local de façon à constituer un fonds d'animaux permettant d'obtenir des surplus de génisses aptes à créer de nouveaux noyaux d'élevage taurin ;

b) l'opération «Elevage zébu » qui a pour 
but de fixer dans la zone forestière de la Palé des groupes d'éleveurs Peul qui, avec leurs troupeaux transhument à travers le pays, pour en obtenir les meilleurs profits en viande;

B) Dans le centre de la Côte-d'Ivoire (régions de Bouaké, Bouaflé, Dimbokro...) plusieurs projets se proposent de créer des fermes d'élevage de bovins naisseurs ainsi que des fermes d'embouche sur prairies artificielles, à côté d'élevage de petits ruminants, porcins, volailles...

En outre dans ces mêmes régions centrales, trois ranches sélectionnent et multiplient des N'Dama destinés à fournir des géniteurs améliorés à toutes les opérations d'encadrement ;

- C) Dans le sud du pays (régions d'Abidjan, Aboisso...) où l'élevage était jusqu'ici quasiment absent, un type d'élevage bovin sous palmeraie a été entrepris visant la diffusion de la race Baoulé en zones forestières;

- D) Enfin dans l'ensemble de la Côted'Ivoire, une opération de diffusion de « Noyaux d'élevage » a démarré depuis longtemps. Deux cents de ces noyaux ont été déjà constitués, qui totalisent un effectif de trois mille têtes.

En dépit de ces efforts, les besoins en viande bovine du pays ne seront pas entièrement couverts, même à long terme. C'est pour cela que ces opérations seront suivies d'autres études cherchant de nouvelles formules susceptibles de renforcer les moyens déjà engagés sur le terrain, tels que : l'embouche industrielle, le système visant à faire féconder les femelles hors du territoire, la multiplication des élevages des petites espèces, etc...

La réussite de ces opérations dépendra de la façon dont sera maîtrisée la "grande » pathologie bovine. En Côte-d'Ivoire, la peste bovine a disparu, avec l'extinction en 1972 du dernier foyer constaté dans le nord du pays, à Tehini, alors que les races locales essentiellement constituées de Baoulé et de N'Dama sont trypanotolérantes. Dans ces conditions, ce sont la péripneumonie et la brucellose qui constituent les deux principaux obstacles au développement du cheptel bovin du pays. Si la péripneumonie peut ne pas poser d'importants problèmes tant que les campagnes de vaccination continueront il n'en est pas de même, pour des raisons évidentes, avec la brucellose qui en pose déjà et en posera à long terme de redoutables, ce qui explique et justifie à la fois les études entreprises pour situer au mieux les données du problème ainsi posé.

\section{MATÉRIEL ET MÉTHODES}

\section{Animaux et régions prospectés}

Les animaux fournisseurs de sérums provenaient de parcs sédentaires encadrés par les sociétés d'Etat, des troupeaux d'élevage traditionnel, des parcs de quarantaines destinés au peuplement des ranches et de troupeaux peuplant ces ranches.

La plupart de ces animaux appartiennent aux races N'Dama et Baoulé, avec un effectif réduit de zébus, aucun d'eux n'ayant été vacciné contre la brucellose.

Les effectifs de ces troupeaux sont variables : ils sont d'une dizaine de têtes (région du sud) à de rares troupeaux de trois à quatre cents têtes dans le nord. La moyenne par troupeau est d'environ cent têtes.

Pour faciliter les opérations, les enquêtes ont été faites en s'appuyant sur les directions régionales des services vétérinaires: carte $\mathrm{n}^{\circ} 2$ :

Nord : Korhogo ; ouest Man est : Abengourou ; centre : Bouaké ; centre ouest : Daloa où l'effectif atteint à peine deux mille têtes, et au sud à Abidjan.

\section{Interventions sur le terrain}

Les prélèvements ont été systématiques. En effet, pour les prélèvements effectués par nos services, il n'a pu être obtenu de renseignements complémentaires (avortements, morbidité, mortalité néonatale, etc...) du fait de la réticence des propriétaires d'animaux ou de la mauvaise disposition des responsables des troupeaux pour collaborer avec les équipes d'intervention.

Du fait des précautions prises, les prélèvements sont tous arrivés en bon état aux laboratoires chargés de les traiter : Korhogo pour le nord, Bingerville pour le centre et le sud.

\section{Traitement des prélèvements}

a) Sérologie : les sérums ont été examinés d'emblée par la méthode de séro-agglutination lente (S. A. L.) et par un test au rose Bengale (R. B.). En cas de résultats discordants, il a été fait appel à la fixation du complément (F. C.).

Dans les rares cas où des avortements ou des hygromas ont été constatés et pour lesquels ces trois méthodes ont donné des résultats négatifs nous avons eu recours au test de COOMB (T. C.). 
HAUTE-VOLTA 10,9 p.100 (1954, 1970 I972, 1973)

SENEGAL 13,3 p.100 (1965, 1966, 1971)

GUINEE-BISSAU 15,0 p.100 (1952)

GUINEE 14,5 p.100 (1974)

SIERRA-LEONE 10,4 p.100 (1969)

\section{COTE D'IVOIRE}

10,8 p. $100(1970,1971,1972)$

10,8 P.100 $(1975,1976,1977)$

GHANA 23,5 p.100 (1969)

NIGERIA 8,8 p.100 (1967, 1970, 1972, 1974)

CAMEROUN 10,3 p.100 (1970)

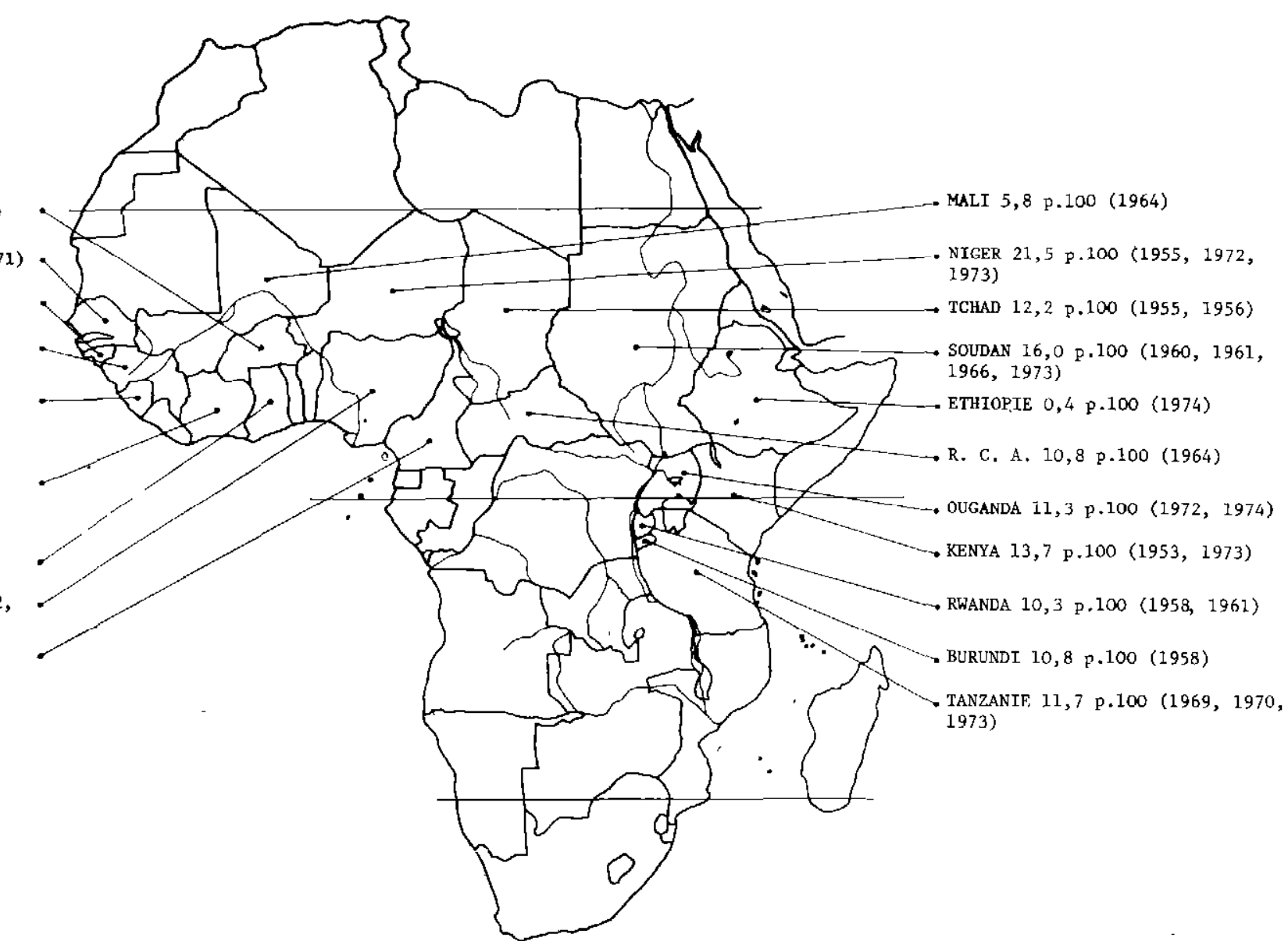

Pourcentage moyen d'infection individuelle, Entre parenthèses : annêe où les enquêtes ont êté effectuêes. 
Toutes ces recherches ont été effectuées à l'aide de réactifs commercialisés par l'Institut Pasteur de Paris ou par l'Institut Mérieux de Lyon, suivant les techniques classiques en la matière.

\section{Bactériologie}

Les liquides prélevés dans les hygromas ont servi pour isoler des souches à l'aide du milieu Brucelle Agar modifié (Mérieux) suivant la technique classique en la matière.

Les germes isolés ont fait l'objet d'une identification primaire et ceux pouvant être rattachés au groupe Brucella ont été adressés au «Centre National de Références des Brucella» à Montpellier, au service du Professeur ROUX pour identification du biotype.

\section{RÉSULTATS}

Les résultats obtenus sont consignés dans cinq tableaux :

- Le tableau $n^{\circ} \mathrm{I}$ indique le taux d'infection des troupeaux par région administrative. Il a été établi en considérant que tout troupeau comportant un seul animal sérologiquement positif a été tenu pour infecté de brucellose;

- Le tableau $\mathrm{n}^{\circ} \mathrm{II}$ donne les résultats obtenus pendant toute la durée de l'enquête, qui s'est déroulée de juin 1975 à décembre 1977.

Les régions du nord de la Côte-d'Ivoire ont pu être profondément étudiées à cause de la densité de la population bovine et de la possibilité d'une collaboration plus étroite entre le laboratoire et les organismes responsables des projets s'occupant de l'élevage ;

- Le tableau $n^{\circ}$ III donne un échantillonnage des enquêtes les plus caractéristiques, ce qui permet de constater le parallélisme qui existe entre la présence d'hygroma et une sérologie brucellique positive ;

- Le tableau $\mathrm{n}^{\circ}$ IV donne les indications sur la répartition géographique des différentes souches de Brucella abortus isolées dans l'ensemble du pays tout en montrant l'intensité de l'infection dans les troupeaux concernés.

Enfin le tableau $n^{\circ} V$ résume l'ensemble des caractères biochimiques et biotypiques fondamentaux des souches isolées.

\section{DISCUSSION}

\section{Taux d'infection}

Pour bien comprendre la situation, il faut distinguer entre le pourcentage des troupeaux infectés, par rapport au nombre total des troupeaux examinés - qui a été établi en considérant comme tel tout troupeau ayant un seul animal sérologiquement positif - et le pourcentage d'animaux positifs examinés dans la même région tout au long de notre enquête.

a) Taux d'infection des troupeaux

TABL. $\mathrm{N}^{\circ} \mathrm{I} \rightarrow$ Taux d'infection des troupeaux.

\begin{tabular}{|c|c|c|c|}
\hline \multirow{2}{*}{$\begin{array}{l}\text { Direction } \\
\text { rêgionale } \\
\text { Services } \\
\text { Vétérinaires }\end{array}$} & \multicolumn{2}{|c|}{ Troupeaux } & \multirow{2}{*}{$\begin{array}{l}\text { p. } 100 \\
\text { positifs }\end{array}$} \\
\hline & examinës & positifs & \\
\hline $\begin{array}{l}\text { Nord } \\
\text { Korhogo }\end{array}$ & 68 & 68 & 100,0 \\
\hline $\begin{array}{l}\text { Oues L } \\
\text { Man }\end{array}$ & 11 & 9 & $8], 8$ \\
\hline $\begin{array}{l}\text { Est } \\
\text { Abengourou }\end{array}$ & 6 & 6 & 100,0 \\
\hline $\begin{array}{l}\text { Centre } \\
\text { Bouaké }\end{array}$ & 16 & 12 & 75,0 \\
\hline $\begin{array}{l}\text { Centre-ouest } \\
\text { Daloa }\end{array}$ & & en reçu & \\
\hline $\begin{array}{l}\text { Sud } \\
\text { Abidjan }\end{array}$ & 64 & 29 & 45,3 \\
\hline
\end{tabular}

Ce tableau indique que le taux d'infection des troupeaux atteint 100 p. 100 dans la zone dense de Korhogo et dans la région d'Abengourou. En particulier dans la première où se concentrent les plus grands rassemblements, pas un parc, pas un troupeau visité n'est indemne. Suivent les régions de Man et de Bouaké. Par contre les régions du sud (Abidjan) avec des effectifs plus restreints et plus dispersés accusent les plus faibles taux. Cette disparité en soi, en même temps qu'elle révèle l'évolution de la maladie, reflète en quelque sorte l'organisation de l'élevage. C'est le phénomène de focalisation dont parlent CHANTAL et FERNEY.

\section{b) Taux d'infection individuel}

Il est donné par région et au niveau des villages dans le tableau $n^{0}$ II, suivant : 
TABL, N ${ }^{\circ}$ II-Résultats globaux des analyses sêrologiques.

\begin{tabular}{|c|c|c|c|c|}
\hline $\begin{array}{l}\text { Direction régionale } \\
\text { Services vétérinaires }\end{array}$ & Régions prospectêes & $\begin{array}{l}\text { Total animaux } \\
\text { examinés }\end{array}$ & Positifs & Pourcentage \\
\hline $\begin{array}{l}\text { Nord } \\
\text { Korhogo }\end{array}$ & $\begin{array}{l}\text { Boundia1i } \\
\text { Badikaha - Ferke } \\
\text { Korhogo (Nord, Quest, Est) } \\
\text { Kaforo } \\
\text { Katia } \\
\text { Sologo } \\
\text { Tiere } \\
\text { Madinani-Odienne } \\
\text { M'Bengue } \\
\text { Sirasso } \\
\text { Tengrela } \\
\\
\text { Total........ }\end{array}$ & $\begin{array}{r}170 \\
166 \\
4497 \\
121 \\
187 \\
59 \\
382 \\
946 \\
97 \\
96 \\
122 \\
6843\end{array}$ & $\begin{array}{r}16 \\
3 \\
616 \\
85 \\
40 \\
27 \\
109 \\
33 \\
18 \\
9 \\
9 \\
965\end{array}$ & $\begin{array}{r}9,41 \\
1,80 \\
13,60 \\
34,84 \\
\\
3,48 \\
18,55 \\
9,37 \\
7,37 \\
14,10\end{array}$ \\
\hline $\begin{array}{l}\text { Ouest } \\
\text { Man }\end{array}$ & I'ouba & 1793 & 18 & 1,00 \\
\hline $\begin{array}{l}\text { Est } \\
\text { Abengourou }\end{array}$ & $\begin{array}{l}\text { Doropo } \\
\text { Bouna } \\
\text { Bondougou } \\
\qquad \text { Totdl....... }\end{array}$ & $\begin{array}{r}114 \\
191 \\
117 \\
1422\end{array}$ & $\begin{array}{r}10 \\
15 \\
5 \\
30\end{array}$ & $\begin{array}{r}8,77 \\
7,85 \\
4,27 \\
17,10\end{array}$ \\
\hline $\begin{array}{l}\text { Centre } \\
\text { Bouakê }\end{array}$ & $\begin{array}{l}\text { Jouahé et alentours } \\
\text { Dianra } \\
\text { Raviart } \\
\text { Toumondi } \\
\qquad \\
\qquad \text { Total............ }\end{array}$ & $\begin{array}{r}677 \\
59 \\
176 \\
\quad 88 \\
1000\end{array}$ & $\begin{array}{r}53 \\
6 \\
43 \\
10 \\
109\end{array}$ & $\begin{array}{r}7,82 \\
10,16 \\
24,43 \\
13,96 \\
10,90\end{array}$ \\
\hline \multirow[t]{2}{*}{$\begin{array}{l}\text { Centre-Ouest } \\
\text { Daloa }\end{array}$} & Rien reçu & & & \\
\hline & $\begin{array}{l}\text { Aboisso } \\
\text { Bingerville + Anyama } \\
\text { Divo } \\
\text { Fresco } \\
\text { Jacqueville } \\
\text { Lakota } \\
\text { Tiassalé } \\
\\
\quad \text { Total...... }\end{array}$ & $\begin{array}{r}552 \\
661 \\
236 \\
181 \\
202 \\
102 \\
271 \\
2 \quad 285\end{array}$ & $\begin{array}{r}11 \\
26 \\
36 \\
30 \\
79 \\
0 \\
37 \\
219\end{array}$ & $\begin{array}{l}19,92 \\
39,33 \\
15,25 \\
16,57 \\
39,10 \\
13,58 \\
9,58\end{array}$ \\
\hline
\end{tabular}

Ces données traduisent encore plus sensiblement la marche de la maladie avec la densification des regroupements - sauf dans la région de Lakota où pas un seul des cent deux animaux examinés n'a été reconnu comme positif tous les troupeaux sont infectés de brucellose, quoique à des degrés divers. Particulièrement dans les villages des régions du Nord, l'infection s'implante, s'intensifie et s'étend en tache d'huile.

Très faible dans les parcs villageois, les taux d'infection augmentent dans les parcs à effectif nombreux pour s'établir autour d'une moyenne elle-même changeante: de 9,4 à 34,8 p. 100 . Cette moyenne est parfois influencée par des contingences locales à cause de nouvelles infections ou de nouveaux rassemblements ponctuels d'animaux, plus ou moins permanents. Par exemple, au cours du dernier trimestre 1977, le taux d'infection du Sud de la région de Korhogo, où l'effectif bovin est important, est passé de la moyenne de 13 p. 100 à 34,8 p. 100 , pour sept cent cinquante animaux examinés. Cette augmentation soudaine du taux moyen d'infection est moins le signe d'une aggravation de la situation existante que l'existence d'une focalisation transitoire occasionnée en l'espèce par une opération de tri entreprise par une société d'Etat (SODEPRA) en vue de la constitution de "groupements à vocation de coopérative» d'embouche ou de production laitière dans le sud.

\section{Rapport entre la sérologie et la clinique}

La situation actuelle de la brucellose bovine en Côte-d'Ivoire implique qu'une distinction soit faite entre la brucellose-infection mise en évidence par la sérologie et la bactériologie à 
partir des liquides de ponction des hygromas et la brucellose-maladie qui se traduit par des avortements - ou toute autre symptomatologie atypique ou douteuse, encore mal circonscrite - qui sont on ne peut plus préjudiciables au rendement du troupeau et donc à son développement.

La difficulté sur le terrain consiste justement à évaluer la participation effective des brucelles dans les avortements, recensés ou non. Une enquête de CAMUS (3) effectuée dans la région nord du pays - en commun accord avec le laboratoire de Korhogo qui a assuré le sérodiagnostic - a montré l'existence d'avortements dans deux cent soixante-deux des cinq cent quatorze parcs encadrés prospectés, soit 52 p. 100 , cela pour huit cent cinquante-neuf femelles représentant 3,2 p. 100 du total des animaux faisant l'objet d'un encadrement vétérinaire et zootechnique. Sur deux cent soixantesept vaches ayant avorté au moins une fois, 38 p. 100 des avortements ont été imputés à une étiologie brucellique.

Actuellement, en dehors de ces données, il est très difficile d'avoir une idée même approchée du nombre d'avortements survenus dans les troupeaux non encadrés ainsi que de l'importance réelle des Brucella dans ces avortements. L'approche de ces problèmes ne pourra se poursuivre que par le biais de diagnostics sérologiques et bactériologiques.
TABI, N $N^{\circ}$ III-Correspondance entre la présence c'hygroma, la bactêrılogie et la sírologie.

\begin{tabular}{|c|c|c|c|c|c|}
\hline $\begin{array}{c}\mathrm{N}^{\circ} \\
\text { enquête }\end{array}$ & $\begin{array}{l}\text { Présence } \\
\text { d'hvgroma }\end{array}$ & $\begin{array}{c}\mathrm{SAL} \\
\text { U.I. } / \mathrm{ml}\end{array}$ & $R B$ & FC' & $\begin{array}{l}\text { Bactërio- } \\
\text { logie }\end{array}$ \\
\hline 19 & $\begin{array}{l}+ \\
+\end{array}$ & $\begin{array}{r}480 \\
70\end{array}$ & + & $+1 / 32$ & \\
\hline 20 & + & - & - & $+1 / 32$ & \\
\hline 21 & $\begin{array}{l}+ \\
+ \\
+\end{array}$ & $\begin{array}{r}100 \\
\text { SUP. } 640 \\
\text { SUP. } 640\end{array}$ & $\begin{array}{l}+ \\
+ \\
+\end{array}$ & & \\
\hline 22 & $\begin{array}{l}+ \\
+ \\
+\end{array}$ & $\begin{array}{r}\text { SUP. } 640 \\
\text { SUP. } 640 \\
560\end{array}$ & $\begin{array}{l}+ \\
+ \\
+\end{array}$ & & \\
\hline 23 & $\begin{array}{l}+ \\
+ \\
+ \\
+\end{array}$ & $\begin{array}{r}140 \\
560 \\
- \\
140\end{array}$ & $\begin{array}{l}+ \\
+ \\
+ \\
+\end{array}$ & $+1 / 32$ & \\
\hline 26 & + & - & - & - & \\
\hline 62 & + & - & - & - & - \\
\hline 72 & $\begin{array}{l}+ \\
+ \\
+ \\
+ \\
+\end{array}$ & $\begin{array}{r}140 \\
70 \\
80 \\
140 \\
\text { SUP. } 640\end{array}$ & $\begin{array}{l}+ \\
+ \\
+ \\
+\end{array}$ & $\begin{array}{l}+1 / 32 \\
+1 / 32\end{array}$ & $\begin{array}{l}+ \\
+ \\
+ \\
+ \\
+\end{array}$ \\
\hline 75 & $\begin{array}{l}+ \\
+\end{array}$ & $\begin{array}{c}560 \\
\text { PHEN zane }\end{array}$ & $\begin{array}{l}+ \\
+\end{array}$ & & + \\
\hline 77 & $\begin{array}{l}+ \\
+ \\
+ \\
+ \\
+\end{array}$ & $\begin{array}{r}640 \\
140 \\
50 \\
50 \\
60\end{array}$ & $\begin{array}{l}+ \\
+ \\
+ \\
+\end{array}$ & $\begin{array}{l}+1 / 32 \\
+1 / 32 \\
+1 / 32\end{array}$ & \\
\hline $7 \mathrm{~B}$ & + & $\begin{array}{l}120 \\
100\end{array}$ & $\begin{array}{l}+ \\
+\end{array}$ & & \\
\hline
\end{tabular}

TABL. $N^{\circ}$ IV-Répartition géographique des souches isolées.

\begin{tabular}{|c|c|c|c|c|c|c|}
\hline Localités & $\begin{array}{l}\text { Taille des } \\
\text { ếchantillons }\end{array}$ & $\begin{array}{l}\text { Sêrologie } \\
\text { positive }\end{array}$ & Pourcentage & $\begin{array}{l}\text { Nombre } \\
\text { souches } \\
\text { isolëes }\end{array}$ & $\begin{array}{l}\text { Préférence } \\
\text { des souches }\end{array}$ & $\begin{array}{l}\text { Bruceltax } \\
\text { abortus } \\
\text { biotype }\end{array}$ \\
\hline $\begin{array}{l}\text { Soclo } \\
\text { (Centre) }\end{array}$ & $64 / 150$ & 28 & 43,7 & 1 & 5 & I \\
\hline $\begin{array}{l}\text { Jacquęville } \\
\text { (Sud) }\end{array}$ & $202 / 313$ & 79 & 39,1 & 2 & $\begin{array}{l}11 \\
12\end{array}$ & $\begin{array}{l}\mathrm{I} \\
\mathrm{I}\end{array}$ \\
\hline $\begin{array}{l}\text { Eloka } \\
\text { (Sud) }\end{array}$ & $52 / 189$ & 5 & 9,8 & 2 & $\begin{array}{l}13 \\
14 \\
\end{array}$ & $\begin{array}{l}\text { I } \\
\text { I }\end{array}$ \\
\hline $\begin{array}{l}\text { Toumodi } \\
\text { (Centre) }\end{array}$ & $7 / 212$ & 7 & 100,0 & 3 & $\begin{array}{l}15 \\
16 \\
17 \\
\end{array}$ & $\begin{array}{l}\text { I } \\
\text { I } \\
\text { I }\end{array}$ \\
\hline $\begin{array}{l}\text { Karakoro } \\
\text { (Nord) }\end{array}$ & $203 / 249$ & 28 & 14,0 & 1 & 9 & I \\
\hline $\begin{array}{l}\text { Raviart } \\
\text { (Centre) }\end{array}$ & $67 / 107$ & 25 & 37,3 & 5 & $\begin{array}{l}1 \\
2 \\
4 \\
6 \\
7\end{array}$ & $\begin{array}{l}\text { VI } \\
\text { VI } \\
\text { VI } \\
\text { VI } \\
\text { VI }\end{array}$ \\
\hline $\begin{array}{l}\text { Bouaké-ville } \\
\text { (Centre) }\end{array}$ & $57 / 100$ & 16 & $28,0^{\circ}$ & 2 & $\begin{array}{r}8 \\
10\end{array}$ & $\begin{array}{l}\text { VI } \\
\text { VI }\end{array}$ \\
\hline $\begin{array}{l}\text { Polcaha } \\
\text { (Nord) }\end{array}$ & $35 / 91$ & 4 & 11,4 & 1 & 3 & VI \\
\hline
\end{tabular}




\section{Les hygromas}

Le vocable «hygromas » improprement employé (2), désigne des lésions de teno-synovite, bursites, arthrites ou de périarthrites qui apparaissent en des points divers du corps de l'animal. Avec THIENPONT et collab. (7) nous considérons l'hygroma comme synonyme de la brucellose dans son extériorisation souscutanée. Dans le présent travail, les dix-sept souches isolées l'ont été de ces manifestations.

Ces résultats, ainsi que le parallélisme trouvé entre une sérologie positive et la présence d'hygroma (tabl. $\mathbf{n}^{\circ} \mathrm{JII}$ ), nous incitent de plus en plus à tenir d'emblée comme contaminé tout troupeau dans lequel nous constatons un ou plusieurs animaux porteurs de ces lésions.

La situation au moment des enquêtes s'établit comme suit : cinq cent quatorze parcs regroupant six cent quatre-vingt-quatorze vaches porteuses d'hygromas; parmi ces parcs, 73 p. 100 sont infectés de brucellose; trois hygromas sur cinq ponctionnés permettent d'isoler à partir des liquides soumis à la culture bactériologique, une souche de Brucella; sept liquides sur dix présentaient au test du Rose Bengale une agglutination massive, en grains de sable, très rapide. Le tableau $\mathrm{n}^{\circ}$ III est significatif à cet égard.

Les risques d'extension de l'infection dus à leur présence sont élevés car, selon l'habitude des gardiens des troupeaux, ils sont ponctionnés à l'aide de couteaux passe-partout souillant les objets, les animaux et les hommes.

\section{Bactériologie des hygromas}

Les dix-sept souches isolées et identifiées représentent les deux biotypes prédominants. A signaler qu'il s'agit des premières souches isolées en pathologie animale dans le pays.

Il s'agit de Brucella abortus biotype I et de Brucella abortus biotype VI. Elles ont été trouvées indifféremment dispersées dans des foyers éloignés ou contigus (tabl. $\mathrm{n}^{\mathrm{O}}$ IV) sinon dans le même parc.

Elles présentaient les caractères biochimiques et biotypiques exprimés dans le tableau $n^{\circ} \mathrm{V}$. A signaler la sensibilité très marquée au bactériophage T1 pour les souches biotype I.

\section{CONCLUSION}

La brucellose est sérologiquement diagnostiquée sur les bovins en Côte-d'Ivoire dès 1970.

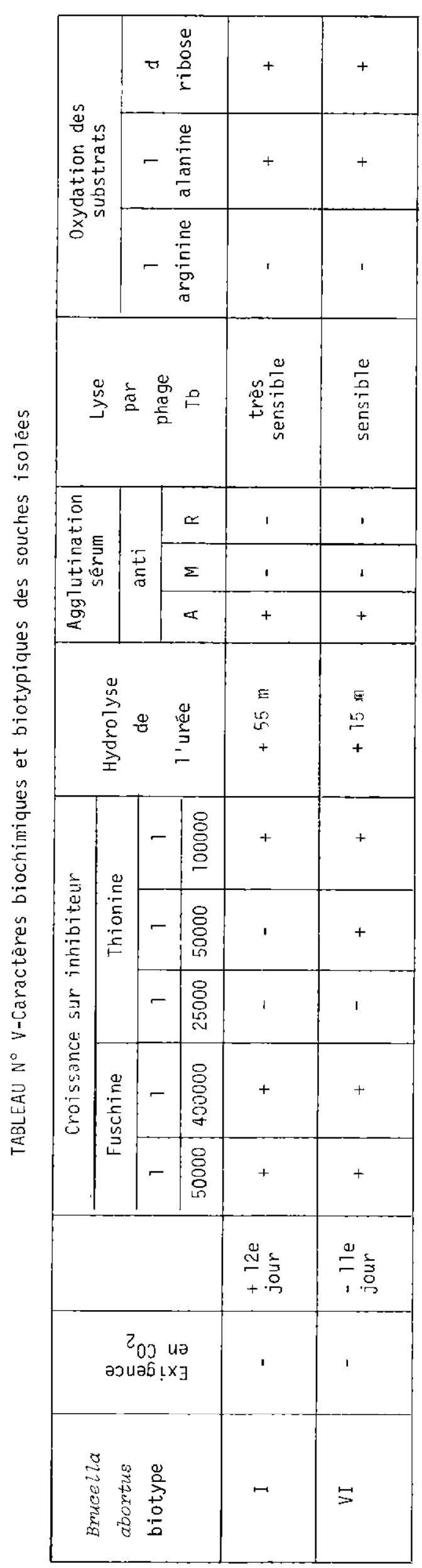


Depuis lors, plusieurs enquêtes menées dans les différentes régions géographiques du pays ont montré que dans certaines régions la maladie est partout présente, particulièrement dans les zones à élevage concentré.

75 p. 100 des troupeaux visités en sont atteints. Le taux d'avortements imputables à Brucella dans certains troupeaux encadrés est de l'ordre de 38 p. 100. D'une région à l'autre, les taux moyens d'infection varient entre 9 et 20 p. 100 avec des variations extrêmes qui peuvent aller presque de 1,09 à 40,00 p. 100 , qui sont parfois le fait de contingences locales particulières (rassemblements, constitution de nouveaux parcs...).

Dix-sept souches de Brucella ont été isolées à partir de liquide de ponction d'hygromas. Cela relie la bactériologie à la clinique en confirmant l'évolution de la brucellose maladie, bien que la symptomatologie, à part la présence d'hygromas et parfois d'avortements, reste encore mal définie.

\section{SUMMARY}

\section{The bovine brucellosis in Ivory Coast. Epidemiology}

An extensive survey covering practically the whole territory of Ivory Coast was carried out. It confirmed the existence of bovine brucellosis in the area studied. It was shown serologically that $10,8 \mathrm{p} .100$ of the twelve thousand three hundred and forty-three sera examined were positive.

A parallel study of eigh hundred and fifty-nine cows showed that miscarrages of brucella origin represented 38 p. 100 of the total. The presence of hygromas in these animals varied considerably.

On the fluids originating from the hygromas seventeen subtypes of brucellosis belonging to the types I and VI were isolated.

\section{RESUMEN}

\section{La brucellosis bovina en Costa de Marfil. Epidemiologia}

Una vasta campaña cubriendo practicamente toda la extension geográfica de Costa de Marfil ha permitido confirmar la existencia de la brucelosis bovina en todo el territorio. Serologicamente, se ha demostrado que de los 12343 sueros sanguineos examinados 10,8 p. 100 son positivos.

En una investigación paralela sobre 859 hembras, los abortos son de origen brucelico en una proporción de 38 p. 100 . La presencia de higromas en estos animales es variable.

De los liquidos de los higromas se han aislado 17 cepas de brucelas perteneciendo a los biotipos I y VI.

\section{BIBLIOGRAPHIE SOMMAIRE (*)}

1. BOHNEL (H.). Recherches sur des causes de mortalité des veaux dans la savane sous-soudanaise dans le nord de la Côte-d'Ivoire. Bull. Epizoot. Afr., 1971, 19 (2) : $145-157$.

2. CHANTAL (J.), FERNEY (J.). La brucellose bovine en Afrique tropicale: quelques aspects cliniques et épidémiologiques, Rev. Méd. vét., 1976, 127 (1) : $19-24$.

3. CAMUS (E.). Bilan des enquêtes sur la brucellose. Propositions SODEPRA. Cellule d'Appui. Décembre 1976.

(*) La bibliographie complète (52 références) sera adressée gratuitement aux lecteurs qui en feront la demande à la Rédaction de la Revue d'Elevage et de Médecine vétérinaire des Pays tropicaux, Centre de Documentation.
4. COULIBALY (F.). La brucellose en Côte-d'Ivoire. Communication à l'O. I. E. Juin 1973.

5. GIDEL (R.), ALBERT (J. P.), LEMAO (G.), RETIF (M.). La brucellose bovine en Afrique occidentale et son incidence sur la santé publique. Résultats de 10 enquêtes épidémiologiques effectuées en Côted'Ivoire, Haute-Volta et Niger de 1970 à 1973. Rev. Elev. Méd. vét. Pays trop., 1974, 27 (4) : 403-418.

6. Rapport sur le fonctionnement pour $1970 \mathrm{du}$ laboratoire national d'élevage de Dakar (Sénégal).

7. THIENPONT (D.), WIKTOR (T.), MORTELMANS (J.), VANDENABELF (G.), BICHE (Y.), FAGARD (P.), PINCKERS (F. R.). Recherches sur la brucellose bovine et humaine au Congo Belge et au RuandaUrundi, à propos d'une enquête dans le territoire d'Astrida (R. U.). Ann. Soc. belge Méd. trop., 1958, 38 : 1049-1056. 